\title{
30 años de historia con los equipos de juniors ITF/ GSDF
}

\author{
Ivan Molina
}

\section{RESUMEN}

Tras treinta años de viajar con el equipo de la ITF quiero hacer un repaso de algunos circuitos, jugadores, resultados en el circuito junior y profesional, y de los países involucrados durante todos estos años, con los diferentes equipos de niños en los cuales participé.
Palabras clave: ITF, GSDF, juniors, equipos

Recibido: 12 Agosto 2017

Aceptado: 25 Octubre 2017

Autor correspondiente:

Ivan Molina.

Correo electrónico:

imolina@aol.com

\section{INTRODUCCIÓN}

\section{Circuito Europeo}

1987 fue el año en el cual la ITF, dirigida por el Director de Desarrollo Doug MacCurdy y con el apoyo de los Torneos de Grand Slam, decidió crear un programa en beneficio de los jugadores junior, con el fin de ayudar a los países menos desarrollados de manera de que sus jugadores tengan exposición internacional a través de la competencia en los mejores torneos del mundo para juniors.

El primer equipo ITF/ GSDF que compitió en Europa contó con la participación de 20 jugadores, 10 niños y 10 niñas; en ellos, un niño y una niña representaban a cada uno de los 10 países sudamericanos. Contábamos con dos entrenadores y un jefe de circuito para todo el equipo.

Hasta 1994, el equipo constaba solamente de jugadores sudamericanos y de un jugador mejicano. En 1995, Dave Miley, nuevo Director de Desarrollo, decidió crear un equipo Internacional que incluyera todos los continentes, de manera que todos los jugadores del mundo tuvieran una oportunidad de ser parte de este programa. También añadió nuevos equipos. El programa continúa así hasta hoy.

Durante los últimos 30 años, un total de 221 jugadores, provenientes de 62 diferentes países, participaron de este programa.

\section{Otros equipos}

Además del Circuito Europeo y a través de los años, el Departamento de Desarrollo de la ITF, con el apoyo del GSDF, creó algunos circuitos diferentes a fin de proporcionar más experiencia y competición a los jugadores.

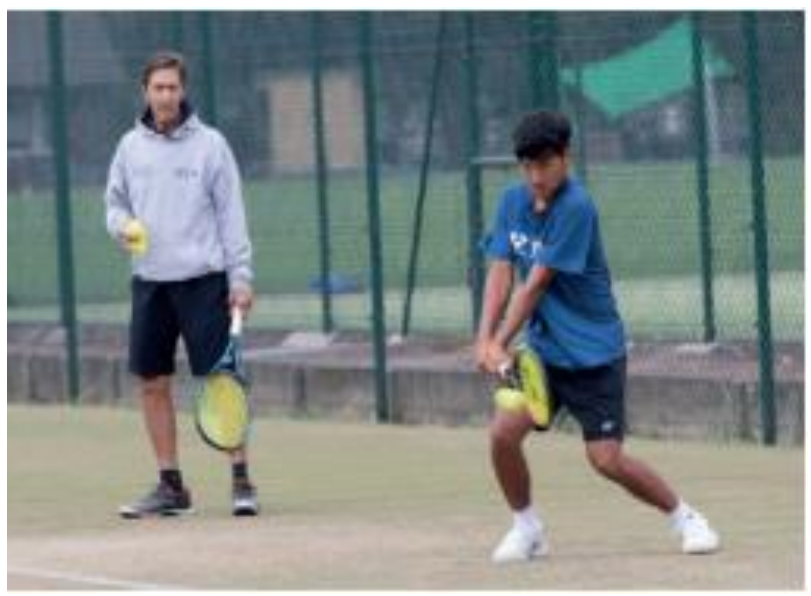

- Circuito de Florida: Este circuito se creó en 1999 y hasta 2002 compitió en torneos como Eddie Herr y el Orange Bowl. Tras un corte, en 2010, se jugó por última vez. Un total de 14 jugadores de 13 países diferentes tuvieron la oportunidad de competir en este circuito.

- Circuito Estados Unidos/ Canadá: Se creó en 1999 y aún continúa en el calendario: 81 jugadores tuvieron la oportunidad de participar en este circuito, estuve con ellos como entrenador, representando a un total de 42 países. 
Luego, entre 2013 y 2015 estuve ausente, y otros entrenadores se ocuparon de un grupo de jugadores. En 2016 fui nuevamente parte del equipo. Un total de 7 nuevos países, no involucrados en otros equipos, estuvieron presentes.

- Circuito de Florida/ Méjico: Este circuito comenzó en 2012 y aún hoy continúa. 12 niños de 12 países diferentes han competido en este circuito.

\section{ALGUNAS ESTADÍSTICAS SOBRE EL RENDIMIENTO DE LOS MEJORES NIÑOS}

Títulos de Individuales en Grand Slam o Finalistas en categoría niños:

1993 Wimbledon, finalista con Jimy Zsymansky de Venezuela

1999 Abierto de los Estados Unidos, ganador con Jarkko Nieminen de Finlandia

2001 Australia, ganador con Janko Tipsarevic de la exYugoslavia

2003 Wimbledon, ganador con Florin Mergea de Romania

2003 Australia, ganador con Marcos Baghdatis de Chipre.

2005 Abierto de los Estados Unidos, ganador con Ryan Sweeting, representante de Bahamas

2007 Roland Garros, ganador con Vladimir Ignatik de Bielorusia, también fue finalista en Wimbledon

2007 Abierto de los Estados Unidos, ganador con Ricardas Berankis de Lituania.

Títulos de Dobles en Grand Slam en categoría niños:

1994 Abierto de los Estados Unidos, Nicolas Lapentti de Ecuador

1994 Roland Garros, Gustavo Kuerten/ Nicolas Lapentti

1997 Roland Garros, Jose De Armas/ Luis Horna

1997 Wimbledon, Luis Horna/ Nicolas Massu

1997 Abierto de los Estados Unidos, Nicolas Massu

1998 Roland Garros, Jose de Armas, de Venezuela.

2002 y 2003, Wimbledon, Florin Mergea/ Horia Tecau

2005 Australia, Kim Sun-Yung, de Corea
2008 Roland Garros, ganador con Henri Kontinen/ Cristopher Rungkat, de Finlandia / Indonesia.

2008 Australia y Wimbledon, Hsieh Chen Peng, de Taipei

2009 Australia, Francis Alcántara/Hsieh Chen Peng de Filipinas/

Taipei

2009 Abierto de los Estados Unidos, Hsieh Chen Peng

2010 Dobles en Roland Garros y Abierto de los Estados Unidos, ganador con Duilio Beretta de Perú/ Roberto Quiroz de Ecuador

2017 Australia, Yu Hsiou Hsu de Taipei

2017 Wimbledon, y Abierto de los Estados Unidos, Yu Hsiou Hsu

\section{Otros Títulos en Grados A, 1}

Durante estos años, los niños compitieron en torneos del Circuito Europeo, que generalmente comenzaba en Santa Croce y terminaba en Wimbledon, siempre había en cada uno un campeón de individuales.

Número total de jugadores juniors en los diferentes equipos

326 jugadores niños compitieron en todos los programas en los cuales participé, algunos de ellos fueron seleccionados para participar en las diferentes giras durante el mismo año, y han jugado dos años o más también.

El total de países que tuvo la oportunidad de traer a jugadores a las diferentes giras es de 65 .

\section{MEJOR RENDIMIENTO DE LOS JUGADORES JUNIOR EX ITF/GSDF EN EL CIRCUITOS PROFESIONAL}

- Los jugadores profesionales clasificados en individuales entre los mejores 10: Gustavo Kuerten, Mariano Puerta, Paradon Srichapan, Marcos Baghdatis, Nicolas Massu, Nicolas Lappenti, Janko Tipsarevic.

- Mejores 20: Hernan Gummy, Victor Troicki, Jarkko Nieminen

- Mejores 50: Fernando Meligeni, Luis Horna, Thomas Belucci, Ricardas Berankis. Yen Hsun Lu, Hyed Chung,

- Mejores 100: Mauricio Hadad, Jimy Zsymansky, Luis Herrera, Ramon Delgado, Alejandro Hernandez, Yeu Tzoo Wang, Ryan Sweeting, Gastao Elias, Alejandro Gonzalez, Damir Zumhur., Radu Talbot, Gastao Elias, Ricardo Melo. 


\section{TÍTULOS DE GRAND SLAM}

\section{Individuales como Profesionales}

Gustavo Kuerten ganó el título en Francia tres veces en individuales: 1997, 2000 y 2001. En el 2000 alcanzó el puesto número 1 en la ATP.

Mariano Puerta, de Argentina, fue finalista en Francia en 2005. Su mejor clasificación en la ATP fue el puesto 9 ese mismo año.

Títulos de Dobles en Grand Slam o Finalistas en categoría Profesionales

Wimbledon

2010, 2011 y 2012, Horia Tecau, finalista

2013, Marcelo Melo, finalista

2015, Jean Julian Roger/ Horia Tecau, ganadores

2017, Marcelo Melo, ganador

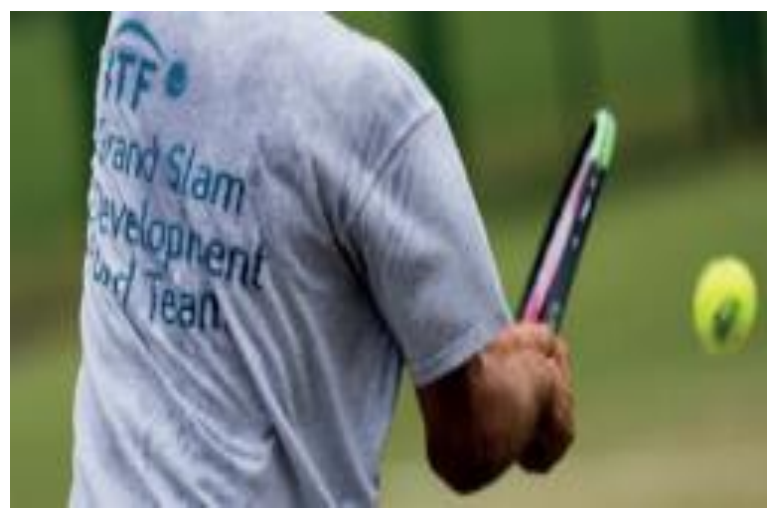

Australia:

2014, Raven Klaasen, finalista

2016, Bruno Soares, ganador

2017, Henri Kontinen, ganador

Roland Garros

2008, Luis Horna, ganador

2015, Marcelo Melo, ganador

Abierto de los Estados Unidos

2010, Aisham UI-Haq Quereshi, finalista

2013, Bruno Soares, finalista
2016, Bruno Soares, ganador

2017, Jean Julian Roger/ Horia Tecau, ganadores

\section{COMPETICIONES DE GRAND SLAM- PROFESIONALES COMPITIENDO}

- Abierto de Australia: Nueve jugadores que estuvieron en Juniors de la ITF anteriormente, compitieron en 2007 en el cuadro principal de individuales. En dobles, 14 jugadores jugaron en el cuadro principal.

- Roland Garros: Un total de 9 jugadores en individuales, que habían sido parte de los equipos junior de la ITF compitieron este año en el cuadro Principal de Roland Garros, todos ellos entre los mejores 100 de la Clasificación de la ATP. En dobles, fueron 15 los jugadores que participaron del equipo ITF.

- Wimbledon: Nueve jugadores en el cuadro de individuales con una clasificación entre los mejores 100 compitieron en este torneo de Grand Slam. Todos ellos formaron parte de ITF/GSDF. En dobles, compitieron 14 jugadores.

- Abierto de los Estados Unidos: Nueve jugadores de individuales compitieron en el cuadro Principal de este Abierto de los Estados Unidos y 14 formaron parte del torneo de dobles. Todos los jugadores de individuales y de dobles fueron parte de los equipos ITF/GSDF.

\section{LOS MEJORES JUGADORES PROFESIONALES CLASIFICADOS EN DOBLES}

Todos los jugadores aquí mencionados fueron parte del equipo ITF y ahora están en la cima de la clasificación profesional de dobles. Melo, Kontinen, Soares, Tecau, Mergea, Aisham Quereshi, Jean Julian Roger, Klaasen, Julio Peralta, Hans Podlipnik. Bruno Soares fue reconocido campeón mundial ITF 2016 en dobles.

\section{OPORTUNIDADES UNIVERSITARIAS}

Entre los beneficios de integrar los equipos ITF/GSDF está la oportunidad que tienen los jugadores de terminar su formación y graduarse gratuitamente, o después de terminar los estudios, tratar de jugar tenis profesional, como ocurrió en varios casos. Muchos miembros de los equipos lograron estas opciones, como vemos en sus historiales.

\section{CONCLUSIÓN}

Quiero agradecer a Doug Maccurdy, Director de Desarrollo en aquel momento, por tener la visión de crear este equipo, y por 
confiar en mí para ser parte de él. Quiero agradecer a la ITF por la oportunidad y confianza que depositaron en mi al hacerme formar parte, y deseo todo lo mejor para el futuro de los próximos equipos. Quiero agradecer al GSDF por su apoyo a este programa, y por brindar a muchos jugadores las oportunidades de cumplir sus sueños. Finalmente, y no menos importante, quiero agradecer a todos los Entrenadores quienes compartieron conmigo esta aventura de ser parte del equipo ITF/ GSDF. Espero que mis contribuciones hayan servido para ayudar a todos los jugadores que integraron esos maravillosos equipos a lograr su mejor potencial, tanto en el tenis como en su vida normal.

CONTENIDO ITF ACADEMY RECOMENDADO (HAZ CLICK ABAJO)

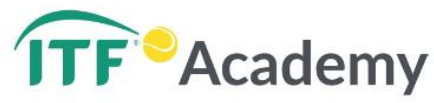

Derechos de Autor (c) 2017 Ivan Molina

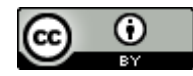

Usted es libre para Compartir -copiar y redistribuir el material en cualquier medio o formato- y Adaptar el documento -remezclar, transformar y crear a partir del material- para cualquier propósito, , incluso para fines comerciales, siempre que cumpla la condición de:

Atribución: Usted debe dar crédito a la obra original de manera adecuada, proporcionar un enlace a la licencia, e indicar si se han realizado cambios. Puede hacerlo en cualquier forma razonable, pero no de forma tal que sugiera que tiene el apoyo del licenciante o lo recibe por el uso que hace de la obra.

Resumendelicencia - Textocompletodelalicencia 